\title{
ON IDEMPOTENTS AND GREEN RELATIONS IN THE ALGEBRAS OF MANY-PLACED FUNCTIONS
}

\section{J. HENNO}

Let $I$ be a non-void set of natural numbers. The union $A_{I}=\bigcup_{i \in I} A_{i}$ of mutually disjoint non-void sets $A_{i}$ is called Menger system [6] if for every $a_{1}, \ldots, a_{m} \in A_{n}$, $b \in A_{m}, n, m \in I$ the element $a_{1} \ldots a_{m} b \in A_{n}$ is uniquely defined and the superassociativity law

$$
a_{1} \ldots a_{m}\left(b_{1} \ldots b_{k} c\right)=\left(a_{1} \ldots a_{m} b_{1}\right) \ldots\left(a_{1} \ldots a_{m} b_{k}\right) c
$$

holds for all $a_{1}, \ldots, a_{m} \in A_{n}, b_{1}, \ldots, b_{k} \in A_{m}, c \in A_{k}, n, m, k \in I$.

Denote by $\varphi_{n}(M)$ the set of all $n$-place functions on the set $M$. Define for every $f_{1}, \ldots, f_{m} \in \varphi_{n}(M), g \in \varphi_{m}(M)$ the function $f_{1} \ldots f_{m} g \in \varphi_{n}(M)$ by

$$
x_{1} \ldots x_{n}\left(f_{1} \ldots f_{m} g\right)=\left(x_{1} \ldots x_{n} f_{1}\right) \ldots\left(x_{1} \ldots x_{n} f_{m}\right) g, \quad x_{1}, \ldots, x_{n} \in M .
$$

For an operation so defined the identity (1) holds, therefore the set $\varphi_{I}(M)=$ $=\bigcup_{i \in I} \varphi_{i}(M)$ is a Menger system, called a full function system (on the set $M$ ). Every subsystem of $\varphi_{I}(M)$ is called a function system (on $M$ ). Every Menger system is isomorphic to some function system [6].

Particular cases of function systems are clone algebras by Cohn [2]. Clone algebras are the function systems $A_{I}$ which for every $n \in I$ contain projectors $\pi_{i}^{n} \in \varphi_{n}(M), i=1, \ldots, n$ (defined by $x_{1} \ldots x_{n} \pi_{i}^{n}=x_{i}$ for every $x_{1}, \ldots, x_{n} \in M$ ). Semigroups and full transformation semigroups are also particular cases $(I=\{1\})$ correspondingly of Menger systems and full function systems.

The aim of the previous paper is to investigate in Menger systems concepts corresponding to idempotents and Green relations in semigroups. Two analogues of regular elements are termed and their properties are found to be similar to the properties of regular elements in semigroups. An analogue of maximal subgroup containing a given idempotent is also discussed.

Call element $e$ of a Menger system an idempotent, if $e \ldots e e=e$. By the above definition and (2) function $f \in \varphi_{n}(M)$ is an idempotent if and only if

$$
x \ldots x f=x
$$

for every element $x$ from the range $M^{n} f$ of the function $f$. 
Call element $a \in A_{n}$ of the Menger system $A_{i}$ weakly regular, if $a=a \ldots$ $\ldots a\left(z_{1} \ldots z_{n} a\right)$ for some $z_{1}, \ldots, z_{n} \in A_{m}, m \in I$. Call $a$ regular, if $a=a \ldots a(z \ldots z a)$ for some $z \in A_{m}, m \in I$. Call a Menger system $A_{I}$ (weakly) regular, if every element of $A_{I}$ is (weakly) regular.

Green equivalences $\mathscr{L}, \mathscr{R}, \mathscr{D}$ and $\mathscr{H}$ were defined for Menger systems in the following way [3]:

$(a, b) \in \mathscr{L}\left(a \in A_{n}, b \in A_{m}, n, m \in I\right)$ if and only if $a=b$ or there exist $s_{1}, \ldots, s_{n} \in A_{m}$, $t_{1}, \ldots, t_{m} \in A_{n}$ such that $a=t_{1} \ldots t_{m} b, b=s_{1} \ldots s_{n} a$;

$(a, b) \in \mathscr{R}$ if and only if $a, b \in A_{n}$ for some $n \in I$ and $a=b$ or there exist $s \in A_{m}$, $t \in A_{k}, m, k \in I$ such that $a=b \ldots b t, b=a \ldots a s$;

$\mathscr{D}=\mathscr{L} \cup \mathscr{R}(=\mathscr{L} \cdot \mathscr{R}$ c.f. [3] $), \mathscr{H}=\mathscr{L} \cap \mathscr{R}$.

Denote by $L(a)(R(a), D(a), H(a))$ the $\mathscr{L}(\mathscr{R}, \mathscr{D}, \mathscr{H})$-class of the element $a$. Define for arbitrary $a \in A_{n}, b \in A_{m}, n, m \in I$ binary relations $\leqq_{L}, \leqq_{R}$, $\varliminf_{H}$ by $a \leqq_{L} b$ if and only if $a=b$ or $a=t_{1} \ldots t_{m} b$ for some $t_{1}, \ldots, t_{m} \in A_{n}$;

$a \leqq{ }_{R} b$ if and only if $a=b$ or $a=b \ldots b t$ for some $t \in A_{k}, k \in I$ (clearly then $n=m$ );

$a \leqq_{H} b$ if and only if $a \leqq_{L} b$ and $a \leqq_{R} b$.

All these relations are preorderings [2] and by definitions $(a, b) \in \mathscr{X}$ if and only if $a \leqq_{X} b$ and $b \leqq_{X} a$, where $\mathscr{X}=\mathscr{L}, \mathscr{R}$ or $\mathscr{H}$.

Lemma 1. Let e be an idempotent element of a Menger system $A_{I}$. The element $e$ is a right identity for an element $a$ (i.e. $a=a \ldots a e$ ) if and only if $a \leqq_{L} e, a$ left identity for $a$ (i.e. $a=e . . . e a$ ) if and only if $a \leqq_{R} e$ and a two-sided identity for $a$ if and only if $a \leqq{ }_{H} e$.

Proof. If $a \leqq_{L} e, e \in A_{n}, a \in A_{m}$, then $a=s_{1} \ldots s_{n} e$ for some $s_{1}, \ldots, s_{n} \in A_{m}$ (the case $a=e$ is trivial) and $a \ldots a e=\left(s_{1} \ldots s_{n} e\right) \ldots\left(s_{1} \ldots s_{n} e\right) e=s_{1} \ldots s_{n}(e \ldots e e)=s_{1} \ldots$ $\ldots s_{n} e=a$ by (1). On the other hand, from $a \ldots a e=a$ follows $a \leqq_{L} e$ by the definition. The other assertions follow quite similarly.

Corollary 1. If $e$ is an idempotent, then $e$ is a right identity for L(e), a left identity for $R(e)$ and a two-sided identity for $H(e)$.

Corollary 2. If $e, f$ are idempotents, then $e \leqq_{L} f$ if and only if $e$...ef $=e$, $e \leqq_{R} f$ if and only if $f \ldots f e=e$ and $e \leqq_{H} f$ if and only if $e \ldots e f=f \ldots f e=e$.

Theorem 1. An element of a Menger system is

1) weakly regular, if and only if there is an idempotent in its $\mathscr{L}$-class

2) regular if and only if there is an idempotent in its $\mathscr{R}$-class.

Proof. Let $a \in A_{n}$ be a weakly regular element of a Menger system $A_{I}$, i.e. $a=a \ldots a\left(z_{1} \ldots z_{n} a\right)$ for some $z_{1}, \ldots, z_{n} \in A_{m}, m \in I$. From here $z_{1} \ldots z_{n} a \in L(a)$. Denote $z_{1} \ldots z_{n} a=e$. By (1) $e \ldots e e=\left(z_{1} \ldots z_{n} a\right) \ldots\left(z_{1} \ldots z_{n} a\right)\left(z_{1} \ldots z_{n} a\right)=z_{1} \ldots$ $\ldots z_{n}\left(a \ldots a\left(z_{1} \ldots z_{n} a\right)\right)=z_{1} \ldots z_{n} a=e$, i.e. $e$ is idempotent.

If $a$ is regular, i.e. $a=a \ldots a(z \ldots z a), z \in A_{m}, m \in I$ then for $f=a \ldots a z$ we have 
$f \ldots f a=(a \ldots a z) \ldots(a \ldots a z) a=a \ldots a(z \ldots z a)=a, f \ldots f f=f \ldots f(a \ldots a z)=(f \ldots f a) \ldots$ $\ldots(f \ldots f a) z=a \ldots a z=f$. Thus $f \in R(a)$ is an idempotent.

On the other hand, if there exists an idempotent $e \in L(a)$ or an idempotent $f \in R(a)$, then $e=z_{1} \ldots z_{n} a, f=a \ldots a z$ for some $z_{1}, \ldots, z_{n}, z$. By Corollary $1 a=a \ldots$ $\ldots a e=a \ldots a\left(z_{1} \ldots z_{n} a\right)$ or $a=e \ldots e a=(a \ldots a z) \ldots(a \ldots a z) a=a \ldots a(z \ldots z a)$, thus $a$ is weakly regular or reguĩar.

Corollary 1. An element of a Menger system is weakly regular if and only if its $\mathscr{D}$-class contains an idempotent. Hence if an element of some $\mathscr{D}$-class $D$ is weakly regular, then all elements from $D$ are weakly regular and elements from containing idempotents $\mathscr{R}$-classes are regular.

Proof. Suppose $a \in D$ is weakly regular. Then there exists an idempotent $e \in L(a) \subset D$. All elements from $R(e)$ are regular (therefore also weakly regular). Since $R(e)$ meets every $\mathscr{L}$-class from $D$ [3], every $\mathscr{L}$-class from $D$ contains weakly regular elements, hence by Theorem 1 consists of weakly regular elements and so does also $D$ as a union of its $\mathscr{L}$-classes. In the other direction the assertion is obvious, since every idempotent is regular.

A subsystem $B_{J}$ of a Menger system $A_{I}\left(J \subset I, B_{n} \subset A_{n}\right.$ for every $\left.n \in I\right)$ is called [3] a left ideal, if $I=J$ and $x_{1} \ldots x_{m} y \in B_{n}$ for every $x_{1}, \ldots, x_{m} \in A_{n}, y \in B_{m}, n, m \in I$, a right ideal, if $x_{1} \ldots x_{m} y \in B_{n}$ for every $x_{1}, \ldots, x_{m} \in B_{n}, y \in A_{m}, m \in I$ and

a two-sided ideal, if $B_{J}$ is a left and a right ideal.

Now Theorem 1 can be reworded in a manner always known for semigroups [1]:

Corollary 2. An element a of a Menger system is weakly regular if and only if the left ideal generated by the element a has an idempotent generator. If an element a is regular, then the right ideal generated by the element a has an idempotent generator. Conversely, if the right ideal generated by an element a has an idempotent generator and has a right identity, then the element $a$ is regular.

Proof. The left ideal generated by an element $a L_{a}=\left\{z \mid z \leqq_{L} a\right\}$. By the definition $(a, b) \in \mathscr{L}$ if and only if $L_{a}=L_{b}$. Hence the assertion about weakly regular elements follows directly from the last theorem.

If $a$ has a right identity, i.e. $a=a \ldots a x$ for some $x$ (in particular, if $a$ is weakly regular), then the right ideal generated by the element $a R_{a}=\left\{z \mid z \leqq_{R} a\right\}$ and the assertion follows quite similarly. Notice that if a right identity for $a$ does not exist, the above expression for $R_{a}$ need not hold.

For arbitrary $f \in \varphi_{n}(M), g \in \varphi_{m}(M)$ we have [3]

$(f, g) \in \mathscr{L}$ if and only if $f$ and $g$ have the same range, i.e. $M^{n} f=M^{m} g$;

$(f, g) \in \mathscr{R}$ if and only if $n=m$ and $f$ and $g$ have the same partition (partition $\tau(h)$ corresponding to the function $h \in \varphi_{k}(M)$ is an equivalence on $M^{k}$ defined by $(\bar{x}, \bar{y}) \in \tau(h)$ if and only if $\left.\bar{x} h=\bar{y} h, \bar{x}, \bar{y} \in M^{k}\right)$;

$(f, g) \in \mathscr{D}$ if and only if the ranges of $f$ and $g$ have the same cardinality and $(f, g) \in \mathscr{H}$ if and only if $f$ and $g$ have the same range and the same partition. 
Call equivalence $\tau \subset M^{n}$ diagonal if every $\tau$-class contains at least one element of the form $(x \ldots x), x \in M$. From (3) it is obvious that a function $f \in \varphi_{n}(M)$ is idempotent if and only if the partition $\tau(f)$ is diagonal and for every $x \in M^{n} \bar{x} f=y$, where $\bar{x}$ and $(y \ldots y)$ belong to the same $\tau(f)$-class. Since $(f, g) \in \mathscr{R}$ if and only if $\tau(f)=\tau(g)$, from the above theorem follows

Corollary 3. A function $f$ is regular if and only if the partition $\tau(f)$ is diagonal.

Theorem 2. The full function system $\varphi_{n}(M)$ is weakly regular.

Proof. Let $g \in \varphi_{n}(M)$. Let $f \in \varphi_{n}(M)$ be such that

1) $M^{n} f=M^{n} g$

2) $(x \ldots x) f=x$ for every $x \in M^{n} g$

Because of 1) $(f, g) \in \mathscr{L}$, because of 2) $f$ is an idempotent.

Corollary. Every Menger system can be embedded into a weakly regular Menger system generated by idempotents.

Proof. For $I=\{1\}$, i.e. for semigroups the assertion was established in [7]. If $I \neq\{1\}$, Menger system $A_{I}$ can be embedded into a weakly regular Menger system $\varphi_{I}(M)$ [6] and $\varphi_{I}(M)$ is generated by idempotents [5].

Call elements $b_{1}, \ldots, b_{n} \in A_{m}$ weakly inverses of an element $a \in A_{n}, n, m \in I$, if $a \ldots a\left(b_{1} \ldots b_{n} a\right)=a, b_{1} \ldots b_{n}\left(a \ldots a b_{i}\right)=b_{i}, i=1, \ldots, n$. Call elements $a \in A_{n}, b \in A_{m}$ inverses (of each other), if $a \ldots a(b \ldots b a)=a, b \ldots b(a \ldots a b)=b$.

Theorem 3. An element of a Menger system has weakly inverses if and only if it is weakly regular and inverses if and only if it is regular.

Proof. If an element has (weakly) inverses then by the above definition it is weakly regular. On the other hand, let element $a \in A_{n}$ be weakly regular, i.e. $a=$ $=a \ldots a\left(z_{1} \ldots z_{n} a\right)$ for some $z_{1}, \ldots, z_{n} \in A_{m}, \quad m \in I$. Let $b_{i}=z_{1} \ldots z_{n}\left(a \ldots a z_{i}\right), \quad i=$ $=1, \ldots, n$. Then $a \ldots a\left(b_{1} \ldots b_{n} a\right)=a \ldots a\left(\left(z_{1} \ldots z_{n}\left(a \ldots a z_{1}\right)\right) \ldots\left(z_{1} \ldots z_{n}\left(a \ldots a z_{n}\right)\right) a\right)=$ $=a \ldots a\left(\left(a \ldots a z_{1}\right) \ldots\left(a \ldots a z_{n}\right) a\right)=a \ldots a\left(z_{1} \ldots z_{n}\left(a \ldots a\left(z_{1} \ldots z_{n} a\right)\right)\right)=a \ldots a\left(z_{1} \ldots z_{n} a\right)=$ $=a, b_{1} \ldots b_{n}\left(a \ldots a b_{i}\right)=\left(z_{1} \ldots z_{n}\left(a \ldots a z_{1}\right)\right) \ldots\left(z_{1} \ldots z_{n}\left(a \ldots a z_{n}\right)\right)\left(a \ldots a\left(z_{1} \ldots z_{n}\left(a \ldots a z_{i}\right)\right)\right)=$ $=z_{1} \ldots z_{n}\left(\left(a \ldots a z_{1}\right) \ldots\left(a \ldots a z_{n}\right)\left(\left(a \ldots a\left(z_{1} \ldots z_{n} a\right)\right) \ldots\left(a \ldots a\left(z_{1} \ldots z_{n} a\right)\right) z_{i}\right)\right)=z_{1} \ldots z_{n}(a \ldots$ $\left.\ldots a\left(z_{1} \ldots z_{n}\left(a \ldots a z_{i}\right)\right)\right)=z_{1} \ldots z_{n}\left(\left(a \ldots a\left(z_{1} \ldots z_{n} a\right)\right) \ldots\left(a \ldots a\left(z_{1} \ldots z_{n}\right)\right) z_{i}=z_{1} \ldots z_{n}\left(a \ldots a z_{i}\right)=\right.$ $=b_{i}$, i.e. $b_{1}, \ldots, b_{n}$ are weakly inverses to $a$. The proof for the case when element $a$ is regular is quite similar.

Corollary. An element of a Menger system has weakly inverses if and only if there is an idempotent in its $\mathscr{D}$-class and inverse if and only if there exists an idempotent in its $\mathscr{R}$-class.

Theorem 4. In a weakly regular Menger system each element has regular weakly inverses. 
Proof. Let $b_{1}, \ldots, b_{n} \in A_{m}$ be weakly inverses of an element $a \in A_{n}$ of a weakly regular Menger system $A_{I}, n, m \in I$. Then $e=b_{1} \ldots b_{n} a$ is idempotent and $(e, a) \in \mathscr{L}$. Furthermore, $e \ldots e b_{i}=\left(b_{1} \ldots b_{n} a\right) \ldots\left(b_{1} \ldots b_{n} a\right) b_{i}=b_{1} \ldots b_{n}\left(a \ldots a b_{i}\right)=b_{i}, \quad i=1, \ldots, n$. Since $A_{I}$ is weakly regular for every $b_{i}$ there are $z_{1}, \ldots, z_{m} \in A_{k}, k \in I$ such that $b_{i}=b_{i} \ldots b_{i}\left(z_{1} \ldots z_{m} b_{i}\right)$. Let $y=z_{1} \ldots z_{m} e$. Then $b_{i} \ldots b_{i}\left(y \ldots y b_{i}\right)=b_{i} \ldots b_{i}\left(\left(z_{1} \ldots z_{m} e\right) \ldots\right.$ $\left.\ldots\left(z_{1} \ldots z_{m} e\right) b_{i}\right)=b_{i} \ldots b_{i}\left(z_{1} \ldots z_{m}\left(e \ldots e b_{i}\right)\right)=b_{i} \ldots b_{i}\left(z_{1} \ldots z_{m} b_{i}\right)=b_{i}$, i.e. $b_{i}$ is regular.

Theorem 5. Let e, $f$ be idempotents from the same $\mathscr{D}$-class of a Menger system $A_{I}$. For every $a \in L(f) \cap R(e)$ there exists an element $b \in R(f) \cap L(e)$ such that $a, b$ are inverses of each other and $a \ldots a b=e, b \ldots b a=f$.

Conversely, if elements $a, b$ are inverses of each other, then the elements $e=a \ldots a b$, $f=b \ldots b a$ are idempotents and $e \in R(a) \cap L(b), f \in L(a) \cap R(b)$.

Proof. Let $a \in L(f) \cap R(e)$, where $e \in A_{n}, f \in A_{m}$ are idempotents of a Menger system $A_{I}$. Then $e=a \ldots a s, f=s_{1} \ldots s_{n} a$ for some $s_{1}, \ldots, s_{n} \in A_{m}, s \in A_{k}, m, k \in I$ and $e \ldots e a=a \ldots a f=a$ by Lemma 1 . Denote $b=f \ldots f(s \ldots s e)$. Now $a \ldots a b=a \ldots a(f \ldots$ $\ldots f(s \ldots s e))=(a \ldots a f) \ldots(a \ldots a f)(s \ldots s e)=a \ldots a(s \ldots s e)=(a \ldots a s) \ldots(a \ldots a s) e=$ $=e \ldots e e=e, \quad b \ldots b a=(f \ldots f(s \ldots s e)) \ldots(f \ldots f(s \ldots s e)) a=f \ldots f((s \ldots s e) \ldots(s \ldots s e)) a=$ $=f \ldots f(s \ldots s(e \ldots e a))=f \ldots f(s \ldots s a)=\left(s_{1} \ldots s_{n} a\right) \ldots\left(s_{1} \ldots s_{n} a\right)(s \ldots s a)=s_{1} \ldots$ $\ldots s_{n}((a \ldots a s) \ldots(a \ldots a s) a)=s_{1} \ldots s_{n}(e \ldots e a)=s_{1} \ldots s_{n} a=f$. Hence $a \ldots a(b \ldots b a)=a \ldots$ $\ldots a f=a, b \ldots b(a \ldots a b)=b \ldots b e=(f \ldots f(s \ldots s e)) \ldots(f \ldots f(s \ldots s e)) e=f \ldots$

$\ldots f(s \ldots s(e \ldots e e))=f \ldots f(s \ldots s e)=b$, i.e. $a, b$ are mutually inverses. From $b \ldots b e=$ $=b, a \ldots a b=e$ follows $(e, b) \in \mathscr{L}$, from $b \ldots b a=f, f \ldots f b=b$ follows $(b, f) \in \mathscr{R}$.

On the other hand, let $a, b$ be inverses, $a \ldots a(b \ldots b a)=a, b \ldots b(a \ldots a b)=b$. Then elements $e=a \ldots a b, f=b \ldots b a$ are idempotens such that $a \ldots a f=a, b \ldots$ $\ldots b e=b$. Hence $(a, f) \in \mathscr{L},(b, e) \in \mathscr{R}$. Furthermore, $a=(a \ldots a b) \ldots(a \ldots a b) a=e \ldots$ $\ldots e a, b=(b \ldots b a) \ldots(b \ldots b a) b=f \ldots f b$, therefore $(a, e) \in \mathscr{R},(b, f) \in \mathscr{L}$. Consequently, $e \in R(a) \cap L(b), f \in L(a) \cap R(b)$.

Theorem 6. Let $a, b$ be elements of a subsystem $B_{J}$ of a Menger system $A_{I}$, $I \subset J$. If the element $b$ is weakly regular in $B_{J}$, then $a \leqq_{L} b$ in $B_{J}$ if and only if $a \leqq_{L} b$ in $A_{I}$.

Proof. It is obvious that from $a \leqq_{L} b$ in $B_{J}$ follows $a \leqq_{L} b$ in $A_{I}$, so we have to prove the contrary.

Let $a \in B_{n}, b \in B_{m}$ and $a \leqq_{L} b$ in $A_{I}$, i.e. $a=t_{1} \ldots t_{m} b$ for some $t_{1}, \ldots, t_{m} \in A_{n}$. Since $b$ is weakly regular in $B_{J}$, there exists an idempotent $e \in B_{k}, k \in J$ such that $(e, b) \in \mathscr{L}$. From here $b \leqq_{L} e$ in $B_{J}$ and since $a \leqq_{L} b, a \leqq_{L} e$ in $A_{I}$. From here $a \ldots a e=a$ by Lemma 1 .

Since $(e, b) \in \mathscr{L}, e=z_{1} \ldots z_{m} b$ for some $z_{1}, \ldots, z_{m} \in B_{k}$. Therefore $\left(a \ldots a z_{1}\right) \ldots$ .. $\left(a \ldots a z_{m}\right) b=a \ldots a\left(z_{1} \ldots z_{m} b\right)=a \ldots a e=a$, i.e. $a \leqq_{L} b$ in $B_{J}$.

By the definition $(a, b) \in \mathscr{L}$ if and only if $a \leqq_{L} b$ and $b \leqq_{L} a$. Consequently, from the above theorem follows 
Corollary. If an element $b$ is weakly regular in a subsystem $B_{J}$ of a Menger system $A_{I}$, then $L_{B}(b)=L_{A}(b) \cap\left(B_{J} \times B_{J}\right)$, where $L_{A}(b), L_{B}(b)$ are the $\mathscr{L}$-classes of the element $b$ in systems $A_{I}, B_{J}$ respectively. Consequently, if a subsystem $B_{J}$ of a Menger system $A_{I}$ is weakly regular, $\mathscr{L}_{B}=\mathscr{L}_{A} \cap\left(B_{J} \times B_{J}\right)$, where $\mathscr{L}_{A}, \mathscr{L}_{B}$ are $\mathscr{L}$-equivalences in systems $A_{I}, B_{J}$ respectively. In particular, $\mathscr{L}_{\varphi_{n}(M)}=\mathscr{L}_{\varphi_{I}(M)} \cap\left(\varphi_{n}(M) \times \varphi_{n}(M)\right)$ for every natural number $n$.

Theorem 7. Let $a, b$ be elements of a Menger system $A_{I}, a \leqq_{L} b$. If $a$ is weakly regular, for every idempotent $e \in L(b)$ there exists an idempotent $f \in L(a)$ such that $f \leqq{ }_{H} e$.

Proof. Let $a \in A_{n}, b \in A_{m}, a \leqq{ }_{L} b$ and let $e \in A_{I}$ be an idempotent from $L(b)$. Since $a \leqq{ }_{L} b, a=t_{1} \ldots t_{m} b$ for some $t_{1}, \ldots, t_{m} \in A_{n}$. Since $e \in L(b), e=z_{1} \ldots z_{m} b$ for some $z_{1}, \ldots, z_{m} \in A_{I}$ and $b \ldots b e=b$. From $a \leqq_{L} b, b \leqq_{L} e$ follows $a \leqq_{L} e$, hence $a \ldots a e=a$ by Lemma 1 .

Since $a$ is weakly regular, $a=a \ldots a\left(x_{1} \ldots x_{n} a\right)$ for some $x_{1}, \ldots, x_{n} \in A_{k} k \in I$ and the element $e^{\prime}=x_{1} \ldots x_{n} a$ is idempotent, $e^{\prime} \leqq{ }_{L} a$. From $a \leqq_{L} e$ follows $e^{\prime} \leqq{ }_{L} e$, hence $e^{\prime} \ldots e^{\prime} e=e^{\prime}$. Denote $e \ldots e e^{\prime}=f$. Then $f \ldots f f=\left(e \ldots e e^{\prime}\right) \ldots\left(e \ldots e^{\prime}\right)\left(e \ldots e e^{\prime}\right)=e \ldots$ $\ldots e\left(\left(e^{\prime} \ldots e^{\prime} e\right) \ldots\left(e^{\prime} \ldots e^{\prime} e\right) e^{\prime}\right)=e \ldots e\left(e^{\prime} \ldots e^{\prime} e^{\prime}\right)=e \ldots e e^{\prime}=f$, i.e. $f$ is idempotent. By the definition $f \leqq_{R} e$. Since $f \leqq_{L} e^{\prime}$, also $f \leqq_{L} e$. Hence $f \leqq_{H} e$. From $e^{\prime} \ldots e^{\prime} f=$ $=e^{\prime} \ldots e^{\prime}\left(e \ldots e e^{\prime}\right)=\left(e^{\prime} \ldots e^{\prime} e\right) \ldots\left(e^{\prime} \ldots e^{\prime} e\right) e^{\prime}=e^{\prime} \ldots e^{\prime} e^{\prime}=e^{\prime}$ follows that $e^{\prime} \leqq{ }_{L} f$. Now from $f \in \leqq_{L} e^{\prime}$ it follows that $\left(f, e^{\prime}\right) \in \mathscr{L}$ and since $e^{\prime} \in L(a)$, also that $f \in L(a)$.

A Menger system $A_{I}$ is called group-like [4] if the only left ideal of $A_{I}$ is $A_{I}$ itself and the only right ideals of $A_{I}$ are $A_{J}=\cup_{n \in J} A_{n}, J \subset I$.

For every $m \in I, a \in A_{m}$ the set $\left\{x \ldots x a \mid x \in A_{n}, n \in I\right\}$ is a left ideal of the Menger system $A_{I}$. Similarly, for every $n, m \in I, a \in A_{n}$ the set $\left\{a \ldots a x \mid x \in A_{m}\right\}$ is a right ideal of the Menger system $A_{I}$. Since every ideal contains ideals of that kind, the above definition is equivalent to the following: a Menger system $A_{I}$ is group-like, if

(4) for every $a \in A_{n}, b \in A_{m}, n, m \in I$ there exists $x \in A_{m}$ such that $x \ldots x a=b$ and

(5) for every $a, b \in A_{n}, n \in I$ there exists $y \in A_{n}$ such that $a \ldots a y=b$.

From (4) it follows that $b \leqq_{L} a$ for every $a, b \in A_{I}$ and because of (5) $b \leqq_{R} a$ for every $a, b \in A_{n}, n \in I$. Consequently, all elements in group-like Menger system $A_{I}$ are $\mathscr{L}$-equivalent and all elements from every $A_{n}$ are $\mathscr{R}$-equivalent (therefore also $\mathscr{H}$-equivalent).

Theorem 8. A Menger system $A_{I}$ is group-like if and only if every $A_{n}, n \in I$ is weakly regular and contains exactly one idempotent.

Proof. From (1) it follows that in every Menger system $A_{I}$ the defined by

$$
a \cdot b=a \ldots a b, \quad a \in A_{n}, \quad b \in A_{m}, \quad n, m \in I
$$

binary operation $\cdot$ is associative. If $A_{I}$ is group-like, by (4), (5) every $\left\{A_{n}, \cdot\right\}$ is 
group, hence contains exactly one idempotent - the identity element $e^{n}$. From (4), (5) it also follows that every $A_{n}$ is weakly regular (in fact, even regular).

On the other hand, let every subsystem $A_{n}, n \in I$ of a Menger system $A_{I}$ be weakly regular and contain idempotent $e^{n}$. Consequently for every $a \in A_{n}, n \in I$ $a \ldots a e^{n}=a$ and $e^{n}=x_{1} \ldots x_{n} a$ for some $x_{1}, \ldots, x_{n} \in A_{n}$. Take $b \in A_{m}, m \in I$ arbitrary. Since $e^{m} \ldots e^{m} a \in A_{m}, e^{m}=z_{1} \ldots z_{m}\left(e^{m} \ldots e^{m} a\right)$ for some $z_{1}, \ldots, z_{m} \in A_{m}$. Therefore, $b=b \ldots b e^{m}=b \ldots b\left(z_{1} \ldots z_{m}\left(e^{m} \ldots e^{m} a\right)\right)=\left(b \ldots b\left(z_{1} \ldots z_{m} e^{m}\right)\right) \ldots\left(b \ldots b\left(z_{1} \ldots z_{m} e^{m}\right)\right) a$ and for $x=b \ldots b\left(z_{1} \ldots z_{m} e^{m}\right)$ (4) holds.

Let $a \in A_{n}, n \in I$. Take $x \in A_{n}$ such that $x \ldots x a=e^{n}$. Then $a=a \ldots a e^{n}=a \ldots$ $\ldots a(x \ldots x a)$ and $(a \ldots a x) \ldots(a \ldots a x)(a \ldots a x)=a \ldots a(x \ldots x(a \ldots a x))=a \ldots$

$\ldots a((x \ldots x a) \ldots(x \ldots x a) x)=(a \ldots a(x \ldots x a)) \ldots(a \ldots a(x \ldots x a) x=a \ldots a x$, i.e. the element $a \ldots a x$ is an idempotent. Hence $a \ldots a x=e^{n}$. For arbitrary $b \in A_{n}$ $a \ldots a(x \ldots x b)=(a \ldots a x) \ldots(a \ldots a x) b=e^{n} \ldots e^{n} b=b$, i.e. for $y=x \ldots x b$ (5) holds also.

For every idempotent $e$ of a semigroup $S$ there is uniquely defined a maximal subgroup of the semigroup $S$, containing $e$ as identity. This will be the set $H(e)$ [1]. In a Menger system $A_{I}$ too with a set $\left\{e^{k}, k \in J \subset I\right\}$ of idempotents, all from one $\mathscr{L}$-class and each from different $A_{k}$ is connected some group-like subsystem of $A_{I}$. For instance, such will be the set $\left\{e^{k}, k \in J\right\}$ itself, since from $\left(e^{k}, e^{l}\right) \in \mathscr{L}$ follows $e^{k} \ldots e^{k} e^{l}=e^{k}$ for every $k, l \in J$. However, there need not exist maximal group-like subsystems, even for only one idempotent.

For example, let the set $M=\{1,2,3\}$. Define functions $e, a, b, c \in \varphi_{2}(M)$ by

\begin{tabular}{cccccc}
$x$ & $y$ & $(x y) e$ & $(x y) a$ & $(x y) b$ & $(x y) c$ \\
\hline 1 & 1 & 1 & 1 & 2 & 2 \\
2 & 1 & 1 & 1 & 2 & 2 \\
2 & 2 & 2 & 3 & 1 & 3 \\
1 & 2 & 2 & 3 & 1 & 3 \\
2 & 3 & 2 & 3 & 1 & 3 \\
3 & 1 & 2 & 3 & 1 & 3 \\
3 & 3 & 3 & 2 & 3 & 1 \\
3 & 2 & 3 & 2 & 3 & 1
\end{tabular}

Functions $e, a, b, c$ have all the same range, equal to $M$. Hence they are all $\mathscr{L}$-equivalent. They also have the same partition $\tau$ (classes of $\tau$ are $\{(11,(21)\}$, $\{(22),(12),(23),(31)\},\{(33),(32)\})$. Thus they all are $\mathscr{R}$-equivalent, and therefore $\mathscr{H}$-equivalent also. The sets $\{e, a\}$ and $\{e, b\}$ are group-like subsystems of the system $\varphi_{2}(M)$, containing an idempotent $e$. However, $e, a, b$ cannot belong together to any group-like subsystem, since $(x y)(a b e)=2$ for every $x, y \in M$, i.e. the range of the function abe is $\{2\}$ and $(e, a b e) \notin \mathscr{L}$. Moreover, the element $c \in H(e)$ cannot belong to any group-like system either, since $(x y)(e c e)=2$ for every $x, y \in M$.

For further investigation of group-like subsystems we quote some results from [3]. 
Let $a, b A_{n}$ be elements from $\mathscr{H}$-class $H$ of a Menger system $A_{I}$. Let $s_{1}, \ldots$ $\ldots, s_{n} \in A_{n}, t \in A_{m}$ be such that $s_{1} \ldots s_{n} a=b, a \ldots a t=b$ (because of $(a, b) \in \mathscr{H}$ such elements exist). Then all mappings $x \rightarrow s_{1} \ldots s_{n} x, x \in H$ (for every $(a, b) \in \mathscr{H}$ ) form a simple transitive group $\Lambda(H)$ of permutations of the set $H$. Similarly all mappings $y \rightarrow y \ldots y t, y \in H$ also form a simple transitive group $\Gamma(H)$ of permutations of $H$. The groups $\Lambda(H), \Gamma(H)$ are anti-isomorphic. If $H^{\prime}$ is another $\mathscr{H}$-class from the containing $H \mathscr{D}$-class $D$, the groups $\Gamma(H)$ and $\Gamma\left(H^{\prime}\right)$ are isomorphic. Therefore the groups $\Gamma(H), H \subset D$ are all isomorphic to some group $G_{D}$. Call $G_{D}$ the Schützenberger group of $D$.

If $\mathscr{H}$-class $H$ contains an idempotent, $\{H, \cdot\}$ is a group isomorphic to the group $G_{D}$. The corresponding isomorphism $\pi_{H}: H \rightarrow G_{D}$ may be considered as the right regular representation of $H$.

Let $H^{\prime}$ be another containing idempotent $f^{\prime} \mathscr{H}$-class from $D$. The mapping $\tau_{H H^{\prime}}: x \rightarrow(x) \tau_{H H^{\prime}}=f^{\prime} \ldots f^{\prime} x, x \in H$ is an isomorphism from the group $\{H, \cdot\}$ to the group $\left\{H^{\prime}, \cdot\right\}$. Furthermore,

$$
\tau_{H H^{\prime}} \pi_{H^{\prime}}=\pi_{H}
$$

and $\tau_{H H^{\prime}} \tau_{H^{\prime} H}$ is the identity mapping on the set $H$.

Let $H$ be containing idempotent $f \mathscr{H}$-class from $\mathscr{L}$-class $L$. Define for every idempotent $e \in L, e \in A_{n}$ a partial (n-1)-place function $\Phi_{e}: H^{n-1} \rightarrow H$ by

$$
\Phi_{e}\left(a_{1} \ldots a_{n-1}\right)=\left\{\begin{array}{l}
f a_{1} \ldots a_{n-1} e, \text { if } f a_{1} \ldots a_{n-1} e \in H \\
\text { undefined otherwise. }
\end{array}\right.
$$

Here $a_{1}, \ldots, a_{n-1} \in H$.

Lemma 2. Let $H, H^{\prime}$ be $\mathscr{H}$-classes from $\mathscr{L}$-class L. Let e, $f, f^{\prime} \in L$ be idempotents, $f \in H, f^{\prime} \in H^{\prime}$. Let $a_{i} \in H, b_{i}=\left(a_{i}\right) \tau_{H H^{\prime}} \in H^{\prime}, i=1, \ldots, n-1$. Then $f a_{1} \ldots$ $\ldots a_{n-1} e \in H$ if and only if $f^{\prime} b_{1} \ldots b_{n-1} e \in H^{\prime}$ and if $f a_{1} \ldots a_{n-1} \in H$ then $\left(f a_{1} \ldots a_{n-1}\right) \tau_{H H}{ }^{\prime}=f^{\prime} b_{1} \ldots b_{n-1} e$.

Proof. Let $f a_{1} \ldots a_{n-1} e \in H$ for some $a_{1}, \ldots, a_{n-1} \in H$. Then $f^{\prime} b_{1} \ldots b_{n-1} e=$ $=f^{\prime}\left(\left(a_{1}\right) \tau_{H H^{\prime}}\right) \ldots\left(\left(a_{n-1}\right) \tau_{H H^{\prime}}\right) e=\left(f^{\prime} \ldots f^{\prime} f\right)\left(f^{\prime} \ldots f^{\prime} a_{1}\right) \ldots\left(f^{\prime} \ldots f^{\prime} a_{n-1}\right) e=f^{\prime} \ldots$ $\ldots f^{\prime}\left(f a_{1} \ldots a_{n-1} e\right)=\left(f a_{1} \ldots a_{n-1} e\right) \tau_{H H^{\prime}} \in H^{\prime}$ (here $f^{\prime} \ldots f^{\prime} f=f^{\prime}$ because of $\left.\left(f, f^{\prime}\right) \in \mathscr{L}\right)$. So from $f a_{1} \ldots a_{n-1} e \in H$ follows $f^{\prime} b_{1} \ldots b_{n-1} e \in H^{\prime}$.

On the other hand, let $f^{\prime} b_{1} \ldots b_{n-1} e \in H^{\prime}$. Since $\tau_{H H^{\prime}} \tau_{H^{\prime} H}$ is the identity mapping on $H, \quad\left(b_{i}\right) \tau_{H^{\prime} H}=a_{i}$ for every $i=1, \ldots, n-1$. Now $f a_{1} \ldots a_{n-1} e=f\left(\left(b_{1}\right) \tau_{H^{\prime} H}\right) \ldots$ $\ldots\left(\left(b_{n-1}\right) \tau_{H^{\prime} H}\right) e=\left(f \ldots f f^{\prime}\right)\left(f \ldots f b_{1}\right) \ldots\left(f \ldots f b_{n-1}\right) e=f \ldots f\left(f^{\prime} b_{1} \ldots b_{n-1} e\right)=$ $=\left(f^{\prime} b_{1} \ldots b_{n-1} e\right) \tau_{H^{\prime} H} \in H$.

By the last lemma the function $\Phi_{e}$ does not depend essentially on the choice of the $\mathscr{H}$-class $H$ from its $\mathscr{L}$-class $L$ and can be considered as (depending on $L$ ) a function $\Psi_{e}^{L}$ on the group $G_{D}$, defined by

$$
\Psi_{e}^{L}\left(a_{1} \ldots a_{n-1}\right)=\left(\Phi_{e}\left(\left(a_{1}\right) \pi_{H}^{-1} \ldots\left(a_{n-1}\right) \pi_{H}^{-1}\right)\right) \pi_{H}
$$


where $a_{1}, \ldots, a_{n-1} \in G_{D}$ and $H$ is an arbitrary $\mathscr{H}$-class from $\left.L^{\prime \prime}=L(e)\right)$, containing an idempotent.

Theorem 8. Let $B_{J}=\bigcup_{n \in J} B_{n}, B_{n} \subset A_{n}$ be a subset of a Menger system $A_{J}$, $J \subset I$. The set $B_{J}$ is group-like subsystem of $A_{I}$ if and only if 1)-3) hold:

1) $B_{J}$ belongs to one $\mathscr{L}$-class $L$ of $A_{I}$ (thus also to one $\mathscr{D}$-class $D$ )

2) for every $B_{n}$ there exists containing an idempotent $e^{n} \mathscr{H}$-class $H_{n}$ of $A_{I}$ such that $B_{n} \subset H_{n}$

3) there exists subgroup $G$ of the Schützenberger group $G_{D}$ such that if we denote $\pi_{H_{n}}=\pi_{n}, \Psi_{e^{n}}^{L}=\Psi_{n}$, then for every $n \in J$

$3.1\left(B_{n}\right) \pi_{n}=G$

3.2 the function $\Psi_{n}$ is everywhere defined on $G$ and $G$ is closed under $\Psi_{n}$.

Proof. Let $B_{j}=\bigcup_{n \in J} B_{n}$ be a group-like subsystem of a Menger sysțem $A_{I}$, $B_{n} \subset A_{n}, J \subset I$. As mentioned above, $\mathscr{L}_{B}$ consists of one $\mathscr{L}_{B}$-class, every $B_{n}$ forms one $\mathscr{H}_{B}$-class and every $B_{n}$ contains an idempotent $e^{n}$. Hence $B_{J}$ belongs also to one $\mathscr{L}_{A}$-class $L$ (and thus to one $\mathscr{D}_{A}$-class $D$ ), every $B_{n}$ is contained in some $\mathscr{H}_{A}$-class $H_{n}$ and idempotent $e^{n}$ belongs to $H_{n}$.

Since $B_{n}$ is subsystem of $A_{I}, e^{n} a_{1} \ldots a_{n-1} e^{n} \in B_{n}$ for every $a_{1}, \ldots, a_{n-1} \in B_{n}$. Thus $\Phi_{n}\left(a_{1} \ldots a_{n-1}\right)=e^{n} a_{1} \ldots a_{n-1} e^{n}$ is always defined on $B_{n}$. By (7) $\Psi_{n}$ is then also always defined on the set $\left(B_{n}\right) \pi_{n}$ and the last set is closed for $\Psi_{n}$. By (4), (5) $\left\{B_{n}, \cdot\right\}$ is a group. Since this group is a subgroup of the group $\left\{H_{n}, \cdot\right\},\left(B_{n}\right) \pi_{n}$ is a subgroup of the group $G_{D}$. By (6) for every $a \in B_{n}(a) \pi_{n}=(a) \tau_{n m} \pi_{m}=\left((a) \tau_{n m}\right) \pi_{m} \subset\left(B_{m}\right) \pi_{m}$, i.e. $\left(B_{n}\right) \pi_{n} \subset\left(B_{m}\right) \pi_{m}$. Similarly $\left(B_{m}\right) \pi_{m} \subset\left(B_{n}\right) \pi_{n}$. Thus $\left(B_{n}\right) \pi_{n}=\left(B_{m}\right) \pi_{m}$ for every $n, m \in J$.

On the other hand, suppose 1)-3) hold for the subset $B_{J}=\cup_{n \in J} B_{n}$, $B_{n} \subset A_{n}, J \subset I$.

Since $\left(B_{n}\right) \pi_{n}=G$ is a group and $\pi_{n}$ is an isomorphism, $\left\{B_{n}, \cdot\right\}$ is also a group. Let $e^{n}$ be its identity. Clearly $e^{n}$ is an idempotent, and from $B_{n} \subset H_{n}$ follows that $e^{n}$ is the idempotent from $H_{n}$ and the functions $\tau_{n m}, \pi_{n}$ are defined for every $n, m \in J$.

Since $e^{n}$ is the identity of the group $B_{n}, e^{n} \cdot x=x \cdot e^{n}=x$ for every $x \in B_{n}$. By 1 ) $\left(e^{n}, e^{m}\right) \in \mathscr{L}$ for every $n, m \in J$, thus $e^{n} \cdot e^{m}=e^{n} \ldots e^{n} e^{m}=e^{n}$.

Denote for arbitrary $a \in B_{n}, n, m \in J a(m)=\left((a) \pi_{n}\right) \pi_{m}^{-1}, a^{-1}(m)=\left(\left((a) \pi_{n}\right)^{-1}\right) \pi_{m}^{-1}$. Clearly $a(n)=a$, denote similarly $a^{-1}(n)=a^{-1}$.

By (6) $\tau_{m n} \pi_{n}=\pi_{m}$ for every $n, m \in J$. Hence $\left(\pi_{m}\right)^{-1}=\left(\tau_{m n} \pi_{n}\right)^{-1}=\pi_{n}^{-1} \tau_{n m}$ and for every $y \in B_{m} \quad y=\left((y) \pi_{m}\right) \pi_{m}^{-1}=\left((y) \pi_{m}\right) \pi_{n}^{-1} \tau_{n m}=e^{m} \ldots e^{m}\left(\left((y) \pi_{m}\right) \pi_{n}^{-1}\right)=e^{m} \cdot y(n)$. Thus for arbitrary $x_{1}, \ldots, x_{m} \in A_{n} x_{1} \ldots x_{m} y=\left(x_{1} \cdot e^{n}\right)\left(x_{1} \cdot\left(x_{1}^{-1} \cdot x_{2}\right)\right) \ldots\left(x_{1} \cdot\left(x_{1}^{-1} \cdot x_{m}\right)\right) \cdot$ $\cdot\left(e^{m} \cdot y(n)\right)=x_{1} \cdot\left(e^{n}\left(x_{1}^{-1} \cdot x_{2}\right) \ldots\left(x_{1}^{-1} \cdot x_{m}\right) e^{i n}\right) \cdot y(n)$. Denote the last expression by $w, \quad\left(x_{1}^{-1} \cdot x_{i+1}\right) \pi_{n}=a_{i}, i=1, \ldots, n-1$. Then by (7) $e^{n}\left(x_{1}^{-1} \cdot x_{2}\right) \ldots\left(x_{1}^{-1} \cdot x_{m}\right) e^{m}=$ $=\Phi_{m}\left(\left(x_{1}^{-1} \cdot x_{2}\right) \ldots\left(x_{1}^{-1} \cdot x_{m}\right)\right)=\left(\Psi_{m}\left(a_{1} \ldots a_{n-1}\right)\right) \pi_{n}^{-1}$ and by $3.2 \Psi_{m}\left(a_{1} \ldots a_{n-1}\right) \in G$ is defined. Since $\pi_{n}$ is isomorphism, $w=(w) \pi_{n} \pi_{n}^{-1}=\left(\left(x_{1} \cdot\left(\Psi_{m}\left(a_{1} \ldots a_{n-1}\right)\right) \pi_{n}^{-1} y(n)\right) \pi_{n} \pi_{n}^{-1}=\right.$ $=\left(\left(x_{1}\right) \pi_{n} \cdot\left(\Psi_{m}\left(a_{1} \ldots a_{n-1}\right)\right) \pi_{n}^{-1} \pi_{n} \cdot(y(n)) \pi_{n}\right) \pi_{n}^{-1}=\left(\left(x_{1}\right) \pi_{n} \cdot \Psi_{m}\left(a_{1} \ldots a_{n-1}\right) \cdot(y(n)) \pi_{n}\right) \pi_{n}^{-1}$. Now $\left(x_{1}\right) \pi_{n}, \Psi_{m}\left(a_{1} \ldots a_{n-1}\right),(y(n)) \pi_{n} \in G$ and $G$ is subgroup of the group $G_{D}$. Con- 
sequently, $\left(x_{1}\right) \pi_{n} \cdot \Psi_{m}\left(a_{1} \ldots a_{n-1}\right) \cdot\left(y(n) \pi_{n}\right) \in G$ also. So $x_{1} \ldots x_{m} y=\left(\left(x_{1}\right) \pi_{n} \cdot \Psi_{m}\left(a_{1} \ldots a_{n-1}\right) \cdot\right.$ $\left.\cdot\left(y(n) \pi_{n}\right)\right) \pi_{n}^{-1} \in B_{n}$. Thus $B_{J}$ is a subsystem of the Menger system $A_{I}$.

For every $a \in B_{n}, \quad b \in B_{m}\left(b \cdot a^{-1}\right) \ldots\left(b \cdot a^{-1}\right) a=b \cdot\left(a^{-1} \ldots a^{-1} a\right)=b \cdot\left(a^{-1} \cdot a\right)=$ $=b \cdot e^{n}=b$, i.e. (4) holds for $B_{J}$. Similarly, for every $a, b \in B_{n} a \ldots a\left(a^{-1} \cdot b\right)=$ $=a \cdot\left(a^{-1} \cdot b\right) a^{-1} \cdot a \cdot b=e^{n} \cdot b=b$, i.e. (5) holds also. Thus the subsystem $B_{J}$ is group-like.

\section{References}

[1] Clifford, A. H., and G. B. Preston: The algebraic theory of semigroups, vol. I. - Mathematical Surveys, No. 7, American Mathematical Society, Providence, R. I., 1961.

[2] Cohn, P. M.: Universal algebra. - Harper \& Row, New York-London, 1965.

[3] Henno, J.: Эквивалентности Грина в системах Менгера. - Tartu Riikl. Ül. Toimetised Vih. 277, 1971, 37-46.

[4] Henno, J.: Групповые системы Менгера. - Tallin. Polütehn. Inst. Toimetised. Seer. A 312, 1971, 95-110.

[5] Henno, J.: On the superposition of associative idempotent functions. - To appear.

[6] Hion, JA. V.: m-apные $\omega$-кольцоиды - Sibirsk. Mat. Ž. 8, 1967, 174--194.

[7] Howie, J. M.: The subsemigroup generated by the idempotents of a full transformation semigroup. - J. London Math. Soc. 41, 1966, 707--716.

University of Turku

Department of Mathematics

SF-20500 Turku 50

Finland

Received 18 May 1976
Tallin Polytechnic Institute

Department of Mathematics

Ehitajate tee 5

Tallin 200016

USSR 